Article

\title{
Evolutionary Multi-Objective Cost and Privacy Driven Load Morphing in Smart Electricity Grid Partition
}

\author{
Miltiadis Alamaniotis * and Nikolaos Gatsis \\ Department of Electrical and Computer Engineering, University of Texas at San Antonio, \\ San Antonio, TX 78201, USA \\ * Correspondence: miltos.alamaniotis@utsa.edu; Tel.: +1-210-458-6237
}

Received: 8 April 2019; Accepted: 21 June 2019; Published: 26 June 2019

check for updates

\begin{abstract}
Utilization of digital connectivity tools is the driving force behind the transformation of the power distribution system into a smart grid. This paper places itself in the smart grid domain where consumers exploit digital connectivity to form partitions within the grid. Every partition, which is independent but connected to the grid, has a set of goals associated with the consumption of electric energy. In this work, we consider that each partition aims at morphing the initial anticipated partition consumption in order to concurrently minimize the cost of consumption and ensure the privacy of its consumers. These goals are formulated as two objectives functions, i.e., a single objective for each goal, and subsequently determining a multi-objective problem. The solution to the problem is sought via an evolutionary algorithm, and more specifically, the non-dominated sorting genetic algorithm-II (NSGA-II). NSGA-II is able to locate an optimal solution by utilizing the Pareto optimality theory. The proposed load morphing methodology is tested on a set of real-world smart meter data put together to comprise partitions of various numbers of consumers. Results demonstrate the efficiency of the proposed morphing methodology as a mechanism to attain low cost and privacy for the overall grid partition.
\end{abstract}

Keywords: load morphing; NSGA-II; smart grid; grid partition; multi-objective optimization; Pareto theory

\section{Introduction}

The introduction of communication and information technologies in the current power infrastructure has accommodated the digital connection of electricity market participants [1] and has enabled their active participation in various activities pertained to grid operation. The concept of an energy Internet as introduced in [2] is a characteristic example of the integration of digital connectivity with power systems. Through active participation, consumers play a significant role in determining the shape of the final load demand, mainly by responding to price signals with their individual demand [3]. In addition, consumers are now exposed to a high volume of information that is utilized for making optimal decisions and fulfilling their goals. Notably, the goals of electricity consumers encompass the maximum fulfillment of energy needs and the minimum possible cost [4].

On one hand, the introduction of digital connectivity in the power grid has leveraged the role of consumers resulting in the enhanced grid stability, minimization of power losses and decreased cost of operation [5]. On the other hand, it came with a reduced degree of consumer privacy given that information sharing and exchange may reveal details about the private life of the consumers. More specifically, load profiles, if shared, can be used to infer the consuming activities of a specific customer. For instance, the use of a washer may be inferred by identifying the consumption pattern of the washer 
in the load profile. Such information may be utilized by third parties for advertising reasons or for nefarious purposes, where burglars may infer the presence or not of the owner and break into the house. Therefore, shared load information comprises of a point of vulnerability that may be used to compromise privacy of consumers in the smart cities of the future [6].

In a market, it is anticipated that consumers are interested in purchasing products of the highest quality at the lowest possible cost. With regard to electricity consumption, electricity consumers care about satisfying their maximum demand while attaining the lowest cost; in other words, they would like to minimize their electricity bill, and fully perform their planned activities [4]. In general, the decision-making process of consumers, including electricity consumers, is a cost driven approach, where the consumer has as a first priority the minimization of the overall cost [7]. In that case, the consumer is prone to morphing his/her load demand in order to retain the cost at a comfortable level. In smart grids, load demand morphing refers to the actions of either cancelling, or shifting the load demand [3] with respect to an initial plan. Cancelling load refers to abandon the scheduling of specific consuming actions, while shifting refers to postponing the consuming actions at a later time, usually at times where the price is lower [8]. The response of consumers with their load demand to prices set by the market operator is known with the general term of "demand response" [9].

There are several approaches that deal with the demand response of consumers aiming at minimizing the cost of consumption. For instance, in [10] an approach that optimizes the electric appliances scheduling for demand response is presented, while in [11] an approach that reduces the load variation limits to minimize consumption costs is introduced. The concept of Virtual Budget as an efficient method for optimizing the electricity cost of demand scheduling using anticipation is proposed in [3], while a sliding window driven method, which utilizes streamed big data for real time electricity consumption optimal adjustment, is proposed and tested in [12]. Furthermore, an optimization algorithm for residential consumption pattern flattening by identifying the time-of-use tariff that minimizes the overall consumption cost is introduced in [13]. In [14], methods that assume the use of interruptible tasks are proposed, whereas in [15] an informatics solution that is based on the synergism of three models in optimizing household appliance management. Furthermore, an autonomous system for demand response that achieves minimal cost for participating in demand response programs consumers is introduced in [16], and a similar approach that assumes consumers response to utility signals prior to any decision making is presented in [17]. Moreover, an approach for scheduling the electricity consumption of a residential community based on the aggregated payment is introduced in [18]. A coordinated approach considering a community of prosumers is discussed is in [19], while the distributed coordination of a grouped consumption using the alternative directions method of multipliers is introduced in [20]. Overall, the variety of demand response approaches aim at securing the operation of the grid [21], while the consumers target to minimize their consumption expenses [22]. However, there are demand response methods that rather focus on consumers' privacy preserving than the cost-minimizing. An example is presented in [23], where a privacy preserving method employs data encryption in the form of a homomorphic encryption of the group aggregated demand. Further, a method focusing on incentive-based demand response of consumers using cryptographic primitives, such as identity-committable signatures and partially blind signatures, is presented and tested in [24]. Data exchange architectures to ensure the privacy of electricity consumption profiles are presented in [25] and [26], where in the first case trusted-platform modules for advanced metering infrastructure (AMI) are used, and in the second case a set of Internet-of-Things tools are put together. In [27], a secure algorithm tailored for bidding driven markets is proposed utilizing cryptographic primitives without any explicit third trusted party. Lastly in [28], a study that involves the use of privacy threat models together with attributed based encryption is provided.

The proposed demand response methods are focusing on single residents and are proposed within the framework of optimizing the power grid infrastructure. Furthermore, they do not exploit the ubiquitous digital connectivity that will be the backbone of the smart cities and smart grids. In this work, we propose an approach that builds upon that connectivity driven future. Notably, we 
see that both the digital connectivity and the smart grid technologies are the enablers for smart cities. In particular, we assume that consumers, which are also residents of a smart city, are able to connect and form "virtually connected groups" [29]. This grouping of the residents/consumers is performed at the cyber level and subsequently allows consumers to form energy partitions within the smart grid [30]. Hence, we will exploit digital connectivity of smart grids to allow consumers within the same partition to collaborate and formulate the partition's demand response. The partition demand response is a single load pattern that coincides with the morphed aggregation of the individual demand patterns of the consumers within the partition, an idea that has been proposed in [31].

In this work, we push further the envelope in load morphing by assuming that the partition demand response results from the concurrent consideration of the partitions consumption expenses and privacy. The paper introduces a new approach where citizens collaborate in order to concurrently attain low cost and high privacy as a group. A premature version of this method was presented in [32], where its high potential for smart grids was highlighted. As compared to [32], this paper presents in more details the morphing method, while it applies it in a higher variety of smart meter data taken from the power grid of Ireland [33]. Notably, the proposed approach considers the morphing problem as a multi-objective problem whose solution is located by an evolutionary algorithm. In this work, the non-dominated sorting genetic algorithm-II (NSGA-II) is adopted to provide a solution to the final morphing of the demand [34]. NSGA-II is a genetic algorithm that utilizes the Pareto optimality theory to identify a solution that optimizes both the cost and privacy of the grid partition [35].

At this point it should be noted, that the current work significantly differs from the works in [29-31]. Those works focus only on enhancing the privacy of the group of the consumers without considering the cost of the final morphed consumption pattern, whereas the current work also explicitly considers the cost of consumption. Furthermore, in References [29,31] a genetic algorithm is adopted to solve a single objective optimization problem, while in the current work the genetic algorithm is selected for locating a solution to a multi-objective problem.

The innovation in the current work is the concurrent consideration of both privacy and consumption cost in a grid partition, and their concurrent optimization through Pareto optimality, which finds the optimal tradeoff between the two objectives, i.e., cost and privacy. This work aspires to show that the morphing of partition consumption driven by those two objectives is a complex optimization problem with multiple constraints, that can be solved by genetic algorithms. Notably, genetic algorithms have the ability to always identify a global optimal solution (or near optimal) to complex problems independently of the number of objectives and constraints.

The roadmap of this paper is as follows: In the next section a brief introduction to evolutionary computing and Pareto optimality is given, while in Section 3 the morphing methodology is presented. In Section 4, the results on a set of real-world datasets are presented and discussed, while Section 5 concludes the paper.

\section{Background}

\subsection{Introduction to Evolutionary Computing}

The set of stochastic optimization techniques whose framework has been inspired by various natural evolution processes are known as "evolutionary computing". Notably, evolutionary computing algorithms are identified as part of the broader class of artificial intelligence algorithms. Some of the most widely used evolutionary algorithms, which have been utilized in various applications, encompass genetic algorithms, genetic programming, swarm intelligence, and artificial immune systems [36].

The main principle upon which evolutionary computing algorithms are built is the selection of an initial population and the subsequent rise of the population fitness by following a natural selection process, based upon the survival of the fittest individuals. Similar to nature, the evolutionary 
computing algorithms select as the final solution the fittest population individuals. In sum, the essential steps that evolutionary algorithms have in common are the following [36]:

- Maintenance of a set of candidate solutions (population);

- Fitness evaluation and sampling of the current population of solutions; and

- Recombination and mutation of solutions to generate new improved solutions.

The above steps drive the implementation of various evolutionary algorithms despite the fact that each one differs in technical details. The outline of a general evolutionary algorithm is depicted in Figure 1.

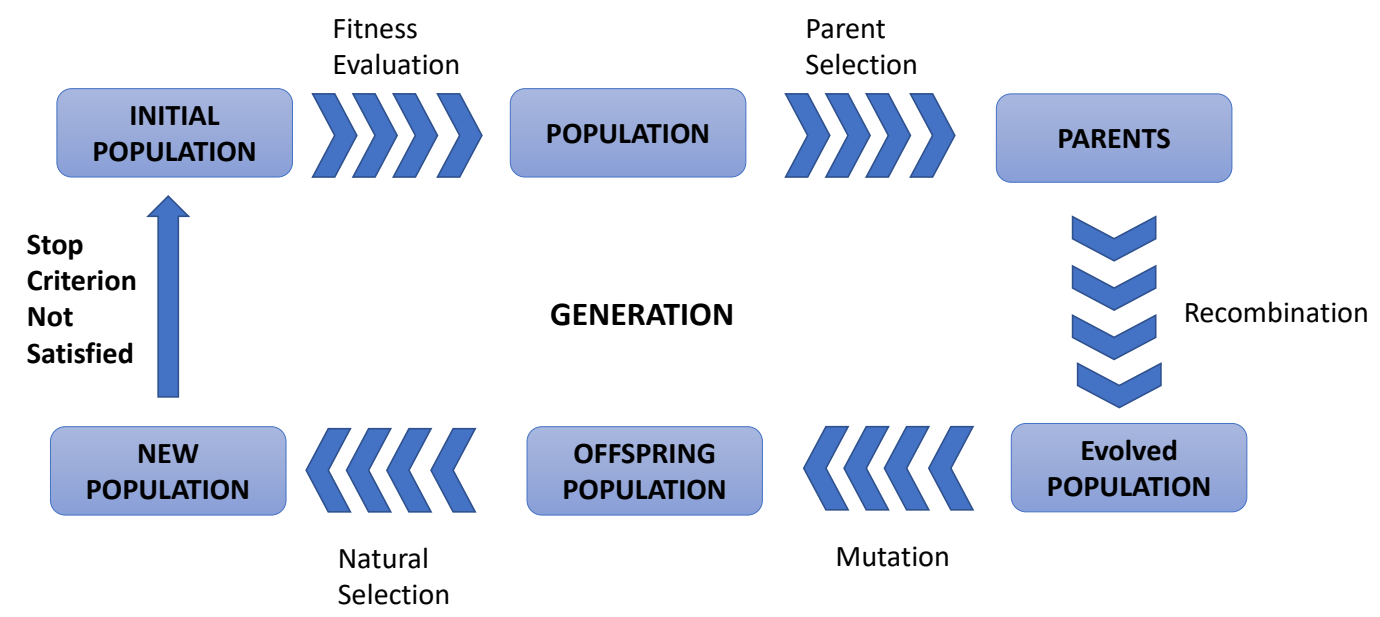

Figure 1. Outline of a general evolutionary computing algorithm.

It should be noted that the full cycle as shown in Figure 1 denotes a generation; thus, the generation is an algorithmic iteration, where transition to a new iteration is initiated when the stopping criterion is not satisfied.

Genetic algorithms are evolutionary algorithms that are used to find solutions in optimization problems that are either single-objective or multi-objective. They are powerful algorithms that identify an optimal solution or a near optimal solution. In addition, they are able to provide solutions to highly complex problems where conventional convex optimization algorithms fail. However, their operation needs the initial determination of a set of variables, i.e., initial population, probability of mutation etc, that may affect their performance. Furthermore, genetic algorithms cover a high area of the search space, but seeking for a solution in a wide area makes them computationally slow. However, their ability of find a global solution promotes them as an attractive option, despite their slow execution time. In this text, the non-dominated sorting algorithm-II will be used for identifying a solution by utilizing the Pareto optimality theory.

\subsection{Pareto Optimality}

Problems that involve the optimization of multiple objectives are called multi-objective optimization problems, or alternatively vector optimization. The multi-objective problems are following general formulation for minimization problems:

$$
\begin{aligned}
\min C(\mathbf{x}) & =\left[C_{1}(\mathbf{x}), C_{2}(\mathbf{x}), \ldots, C_{N}(\mathbf{x})\right] \\
\text { s.t. } \mathrm{f}_{i}(\mathbf{x}) & \leq 0, \quad i=1, \ldots, k \\
\mathrm{~g}_{j}(\mathbf{x}) & =0, \quad j=1, \ldots, m
\end{aligned}
$$


with $C_{\#}()$ denoting an objective function, $N$ being the population of objective functions, $k$ the population of inequality constraints, $m$ the number of equality constraints, and $\mathrm{f}_{\#}()$ and $g_{\#}()$ being valid analytical functions.

As opposed to the single-objective optimization, multi-objective optimization problems typically do have more than a single global solution. As a result, there is a need to determine a set of criteria as to whether a solution is recognized as optimal or not. To that end, the set of criteria that identify a solution as an optimal one are set by the Pareto optimality theory [37]. According to Pareto theory, "a point, $\mathbf{x}^{*} \in \mathbf{X}$, is Pareto optimal iff there does not exist another point, $\mathbf{x} \in \mathbf{X}$, such that $\mathbf{C}(\mathbf{x}) \leq \mathbf{C}\left(\mathbf{x}^{*}\right)$, and $C_{i}(\mathbf{x}) \leq C_{i}\left(\mathbf{x}^{*}\right)$ for at least one function [38]". To make it clearer, a solution is Pareto optimal if there is no other solution that improves at least one objective without adversely affecting any of the rest objectives.

Notably, the population of Pareto solutions pertained to a specific problem can be infinite. The population of the Pareto solutions consist of the Pareto optimal set, while the projection of the Pareto optimal set on the objective space is known as the Pareto frontier [38]. A general illustrative example of Pareto frontier consisting of two objectives is depicted in Figure 2.

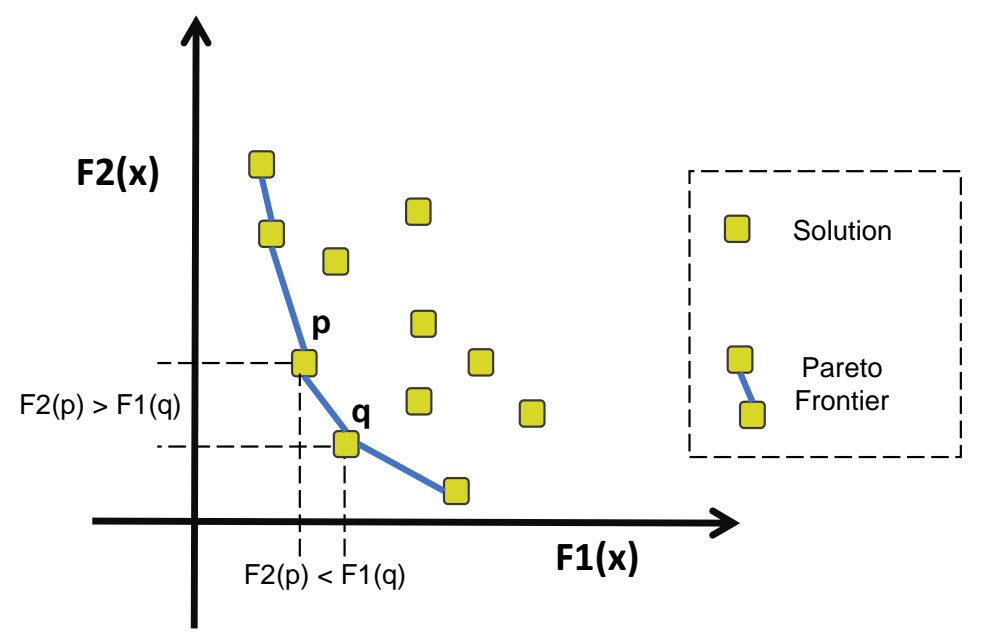

Figure 2. Illustrative example of a Pareto Frontier for a two-objective problem [32].

In addition to the Pareto theory, the concept of a non-dominated or dominated solution is of significance. A solution $\mathbf{x}_{1}$ is identified as dominated over $\mathbf{x}_{2}$ when: (i) The solution $\mathbf{x}_{1}$ is not worse than solution $\mathbf{x}_{2}$ in all objective functions, and (ii) the solution $\mathbf{x}_{1}$ is strictly better than $\mathbf{x}_{2}$ in at least one objective function [38]. If the above conditions are not valid at the same time, then the solution $\mathbf{x}_{1}$ is non-dominated over $\mathbf{x}_{2}$ [38].

\section{Grid Partition Load Morphing Methodology}

In this section, the load morphing of smart grid partitions methodology is presented. It should be noted that the way the partitions are formed in the grid is not of interest to the current manuscript. For instance, one way to form partitions is via grid partitioning as discussed in [39]. The methodology presented in this manuscript formulates a multi-objective problem composed of a dual of objective functions [8]. The two objectives, which express the consumption cost and the degree of privacy in the grid partition respectively, are minimized by seeking an optimal solution to a multi-objective problem. The NSGA-II algorithm is applied to identify an optimal solution. The block diagram of the proposed methodology is presented in Figure 3, where its individual steps are clearly provided. 


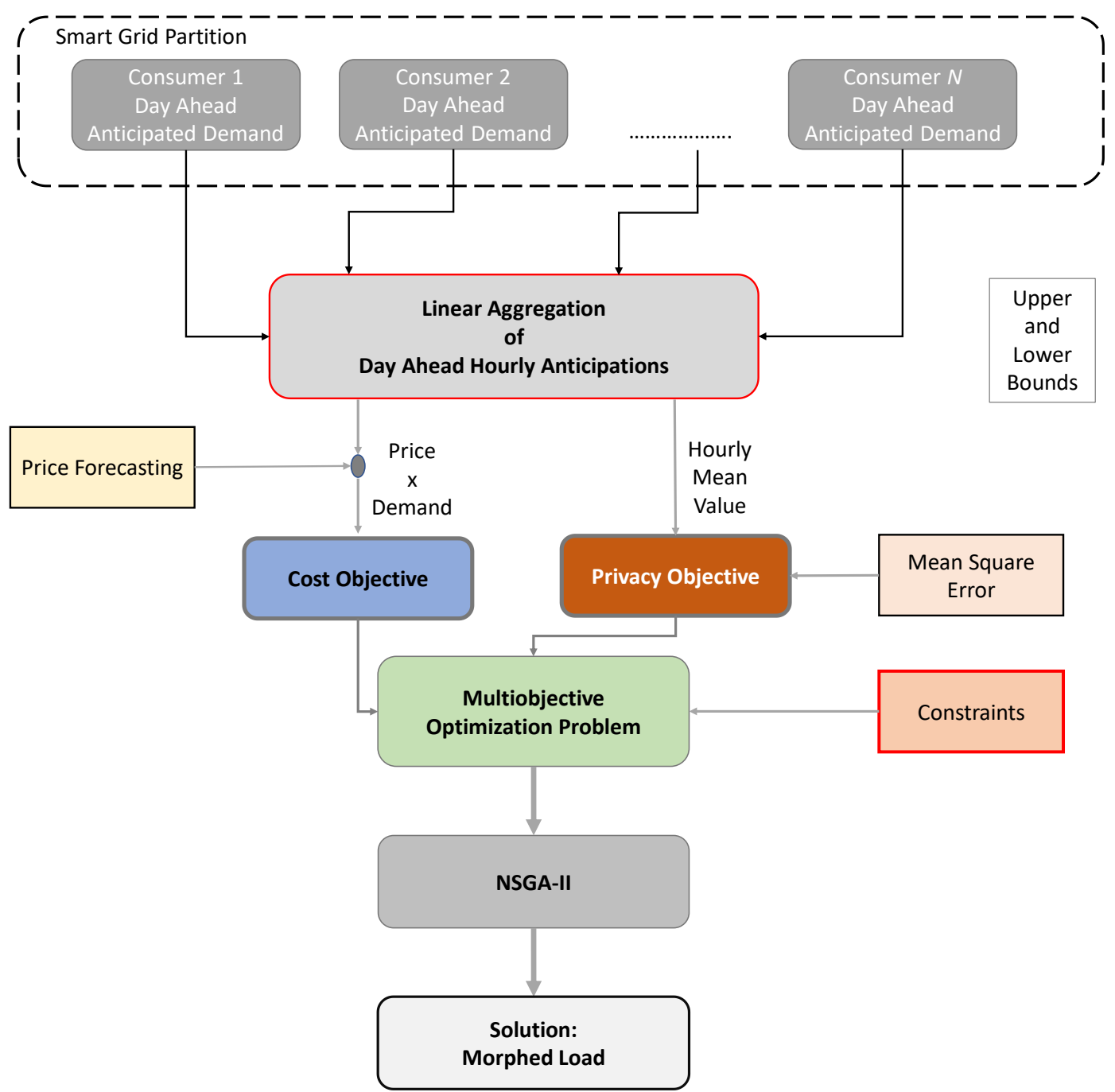

Figure 3. Block diagram of the grid partition load morphing methodology.

Initially, the consumers within the grid partition anticipate their day ahead load demand. The anticipation is conducted at an hourly level, i.e., that means a set of 24 load values for the next day is provided. The overall load anticipation for a whole day is denoted as given below:

$$
\Sigma L=\sum_{i=1}^{24} L_{i}
$$

where $L_{i}$ is the anticipated load at hour $i$. In addition to the anticipated load, the consumers are also providing their hourly upper and lower bounds of their anticipated load denoted as $U_{i}$ and $F_{i}$ for the hour $i$. These bounds coincide with the maximum and minimum values between which the initial anticipation can be morphed for a specific hour, and we call them the "morphing bounds". To make it clearer, when the anticipation for the hour $i$ is $L_{i}$, then the anticipation can be morphed within the tube $\left[L_{i}-F_{i}, L_{i}+U_{i}\right]$.

In the next step, the individual consumers' anticipated loads are aggregated and a single anticipated load demand signal is obtained. Thus, the aggregated pattern expresses the anticipated load of all the partition consumers and is expressed as:

$$
A_{i}=\sum_{j=1}^{N} L_{j}^{i} \quad i=1, \ldots, 24
$$


where $L_{j}^{i}$ is the load of consumer $j$ at hour $i$, and $N$ is the population of consumers. Likewise, the individual morphing bounds are also aggregated providing the respective aggregation morphing bounds:

$$
\begin{gathered}
U_{i}^{A}=\sum_{j=1}^{N} U_{j}^{i} \quad i=1, \ldots, 24 \\
F_{i}^{A}=\sum_{j=1}^{N} F_{j}^{i} \quad i=1, \ldots, 24
\end{gathered}
$$

where $U_{i}^{A}$ and $F_{i}^{A}$ are the upper and lower aggregation morphing bounds for the hour $i$. At this point, we introduce the morphed aggregated load, which is denoted as:

$$
A_{i}^{M}=\alpha_{i} \sum_{j=1}^{N} L_{j}^{i} \quad i=1, \ldots, 24
$$

with $A_{i}^{M}$ being the morphed aggregated load at hour $i$, and $\alpha_{i}$ the morphing factors for the hour $i$. The morphing factors express the degree of morphing, where: (i) $\alpha_{i}=1$ denotes no morphing, (ii) $\alpha_{i}<1$ denotes decreasing of the initial anticipation, and (iii) $\alpha_{i}>1$ denotes increasing of the initial anticipation. Notably, for $\alpha_{i}=1$ Equation (6) drops down to Equation (3).

Once the aggregated values have been computed, then the objective functions are formulated. According to Figure 3, two objectives are formulated, namely, the cost and privacy objectives. The cost objective expresses the daily cost of purchasing the anticipated load and is formulated as:

$$
\text { Cost }=\sum_{i=1}^{24} A_{i}^{M} \cdot P_{i}
$$

with $P_{i}$ being the day ahead forecasted electricity price for the hour $i$.

Formulation of the second objective, which expresses the degree of privacy, is more complex than that of the cost. It should be noted that as a measure of privacy we assume the degree of variance in the load pattern. On one hand a highly varying pattern is a carrier of information that can be easily extracted and subsequently lead to inferences about consumption activities. In other words, variability can be a source of information-the peaks and valleys of the pattern can be associated with consumption activities. On the other hand, a constant load pattern exhibits no variance and thus, inference making becomes challenging. In this work, we aim at deriving a constant load pattern by morphing the aggregated demand. To that end, we adopt a target constant pattern that is equal to the mean value of the aggregated value given below:

$$
M=\frac{1}{24} \sum_{i=1}^{24} A_{i}
$$

where $A_{i}$ is the aggregated value for hour $i$ computed by Equation (3). Furthermore, to quantify the degree of difference between the aggregated load and the mean aggregated value, an error measure is adopted. More specifically, the mean square error is utilized as the objective for expressing the degree of privacy as the distance of the aggregated demand to the mean value. Thus, the privacy objective takes the following form:

$$
\text { Privacy }=\frac{1}{24} \sum_{i=1}^{24}\left(A_{i}^{M}-M\right)^{2}
$$

where $M$ is taken by Equation (8) and $A_{i}$ by Equation (2). 
The objective functions as defined by Equations (7) and (9) are accompanied with a set of constraints. The constraints, which are in the form of box constraints, express the morphing bounds of the aggregated load and are formulated as:

$$
F_{i}^{A} \leq A_{i}^{M} \leq U_{i}^{A} \quad i=1, \ldots, 24
$$

with $A_{i}^{M}$ being the morphed aggregated load (see Equation (6)). As shown in Equation (10), there are 24 box constraints, i.e., one for every hour of the day.

At this point, the two objectives and the respective constraints have been fully defined and therefore, we are able to define the multi-objective problem utilized for load morphing of the grid partition. The particular form of the multi-objective problem is given below:

$$
\left\{\begin{array}{c}
\underset{\alpha_{i}}{\operatorname{minimize}[\text { Cost, Privacy }]} \\
\text { w.r.t. } F_{i}^{A} \leq A_{i}^{M} \leq U_{i}^{A} \\
i=1, \ldots, 24 \\
\text { where Cost }=\sum_{i=1}^{24} A_{i}^{M} \cdot P_{i} \\
\text { Privacy }=\frac{1}{24} \sum_{i=1}^{24}\left(A_{i}^{M}-M\right)^{2} \\
A_{i}^{M}=\alpha_{i} \sum_{j=1}^{N} L_{j}^{i}
\end{array}\right\}
$$

where the optimization process takes the form of a minimization of the two objectives.

The multi-objective minimization problem in Equation (11) is solved utilizing evolutionary computing and more specifically the NSGA-II algorithm. The NSGA-II seeks for non-dominated solutions, which satisfy by default the Pareto optimality criterion. The identified solution which is comprised of a set of 24 optimal morphed values, i.e., optimal $\alpha_{i}^{o p t}, i=1, \ldots, 24$, is the final solution of the problem. Each morphed factor expresses the degree of morphing of the load for the hour $i$. Having computed the morphed values, then the final morphed pattern is obtained by plugging-in the identified solution to Equation (6):

$$
A_{i}^{M}=\alpha_{i}^{o p t} \sum_{j=1}^{N} L_{j}^{i} \quad i=1, \ldots, 24
$$

where the superposition of the morphed values provides the final load curve of the smart grid partition. To conclude, consumers by working all together and exploiting the digital connectivity, may minimize their overall cost and enhance their privacy.

\section{Results}

\subsection{Problem Setup}

The presented methodology is tested on a set of real-world data taken from the Irish power grid [33]. The dataset includes smart meter measurements of various residents. For testing our methodology, we select a set of smart meters and we assume that they belong to the same grid partition. In addition, we assume that the smart meters communicate and are able to apply the presented methodology to form their aggregated load signal. It should be noted, that the final output is the pattern that will be sent out to the utility as its final electricity order. Therefore, by "seizing" the final pattern, the utility as well as any third parties won't be able to make any inferences about the consumption activities of the individual consumers given that their activities will be masked by the morphed aggregated signal. In addition, the overall cost will be low, allowing consumer to "enjoy" 
cheaper consumption in a long-term horizon. Furthermore, ordering and scheduling of an aggregated load demand also allows consumers to perform last minute changes in their demand and exchange those changes without affecting the overall demand. For instance, someone might need to increase his load, while concurrently someone else might have to decrease it by the same amount-a situation that is promoted by the notion of virtual buffers described in $[40,41]$.

In this work, in order to fully define the cost objective the utilization of a forecasted price signal is required. For simplicity, but without compromising the generalization, the forecasted signal is taken with the naïve method [42], which considers as forecasts the observed values of the same day a week ago. The forecasted as well as the real price signal, which the signals were taken from our previous work in [3], are depicted in Figure 4. Furthermore, we need to evaluate the morphing bounds of each consumer's anticipated load. In order to attain a diversity of the morphing values and exhibit a random behavior of the consumers, we implement a randomizer that provides random values of the upper and the lower morphing bounds. The randomizer provides a duet of values sampled from the following intervals:

- $\quad\left[0.7 \times L_{j}^{i}, L_{j}^{i}\right]$ for the lower bound;

- $\left[L_{j}^{i}, 1.3 \times L_{j}^{i}\right]$ for the upper bound.

where $L_{j}^{i}$ is the anticipated load of consumer $j$ for the hour $i$.

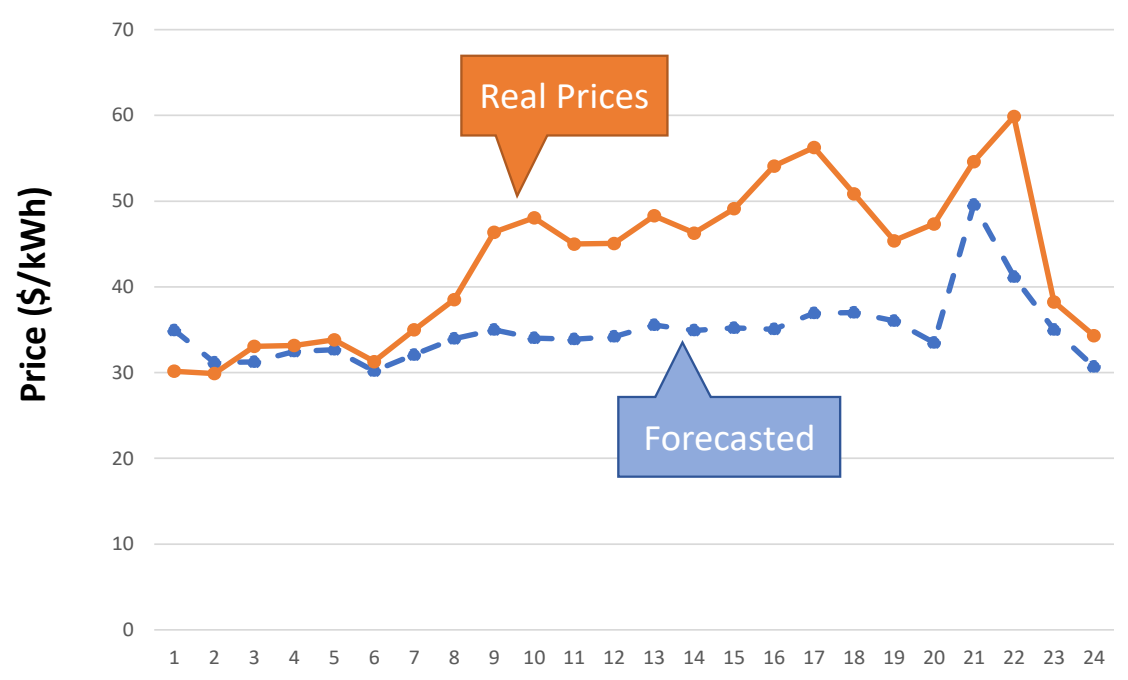

Hour of the Day

Figure 4. Real and forecasted price signal utilized for cost objective.

\subsection{Tested Scenarios}

In this section, the morphing methodology is applied on several scenarios and the respective results are recorded. In particular, we apply the presented methodology on grid partitions comprised six in the first scenario and of seven, 10 and 15 consumers in the rest of the scenarios. The performance of the methodology is assessed in two dimensions: The first dimension compares the overall cost provided by the morphing method to the cost before the morphing takes place. The second dimension measures the degree of privacy attained by the morphing methodology by comparing the correlation coefficients between the load patterns, i.e., between each of the initial individual patterns and the final morphed aggregated pattern. The results concerning the tested scenarios are presented in the subsections given below. 
Scenario 1: Partition of Six Consumers

In this first scenario, we assume a small grid partition comprised of six consumers. Initially, each of the six consumers anticipates his/her day ahead load pattern; the respective load patterns for Consumers 1-6 are depicted in Figure 5. In the next step, the hourly morphing bounds of each of the consumers are obtained; the bounds pertained to the current scenarios are depicted in Figure 6 where both the upper and lower bounds are presented for all six consumers.
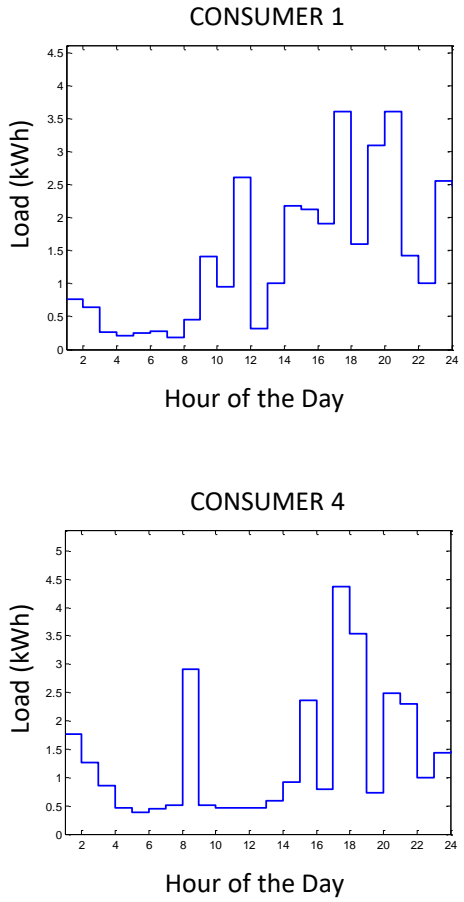
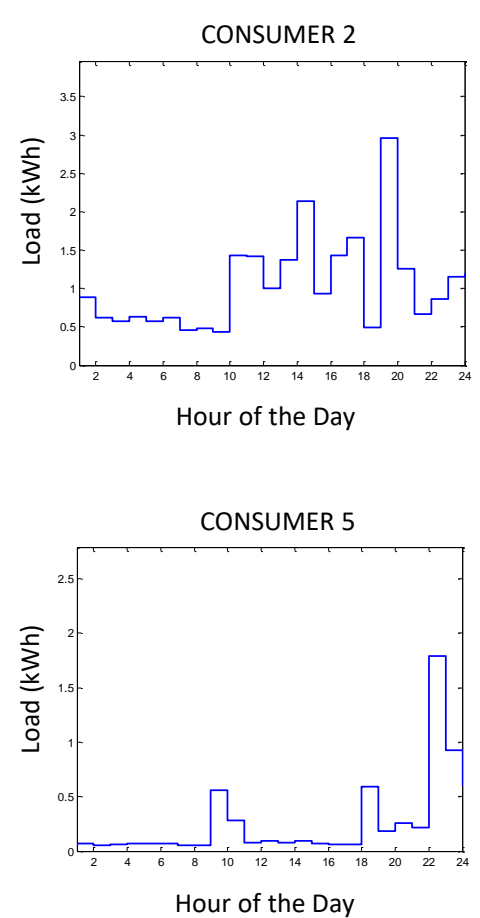
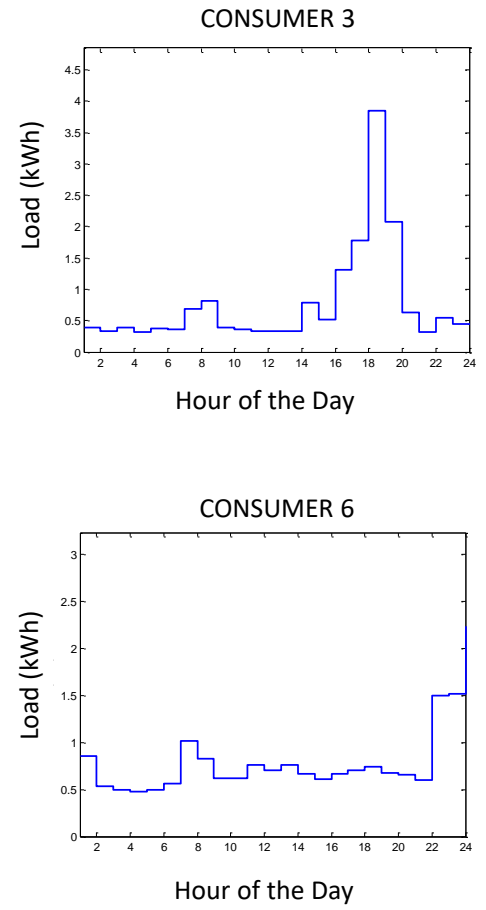

Figure 5. Anticipated load patterns of Consumers 1-6 for test Scenario 1.

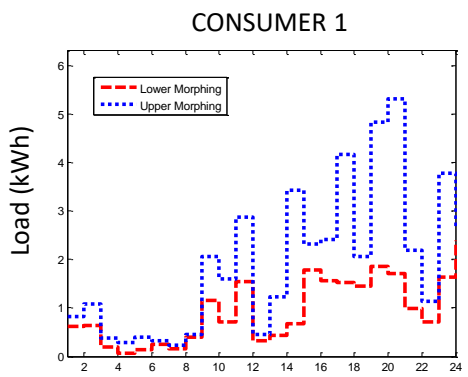

Hour of the Day

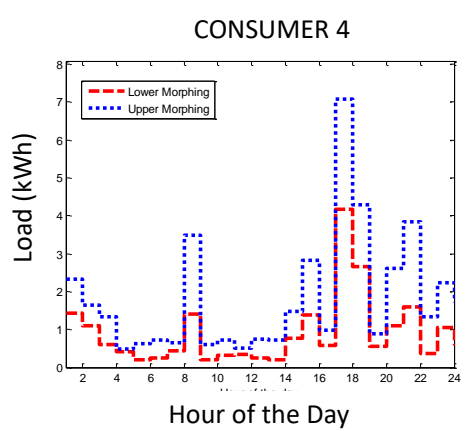

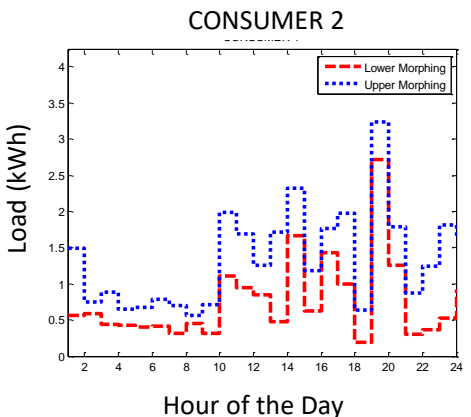
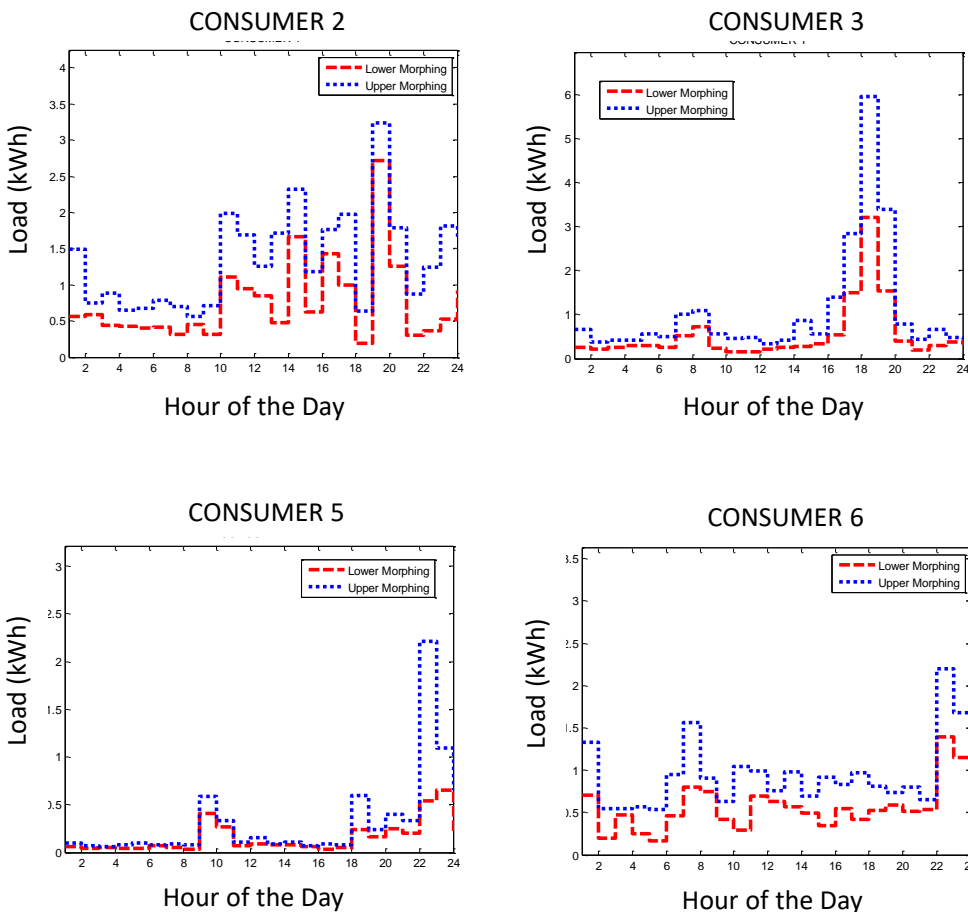

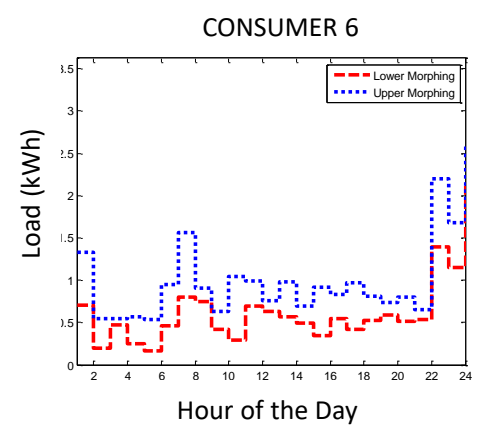

Figure 6. Anticipated load morphing bounds of Consumers 1-6 for test Scenario 1. 
In the next step, the load patterns and their morphing bounds are aggregated to form the partition's load pattern and the respective morphing bounds. The latter ones, will comprise of the box constraints of the optimization problem, whose goal is to minimize the consumption cost as well as minimize the distance of the overall aggregated pattern to the its mean value. The aggregated pattern together with its morphing bounds are presented in Figure 7. In the following step, we compute the mean value of the aggregated pattern that is found to be equal to 5.69. Therefore, we create a constant pattern of the form:

$$
M_{i}=5.69 \quad i=1, \ldots, 24
$$

i.e., a set of 24 values equal to 5.69. The pattern in Equation (13) is used to formulate the Mean Square Error (MSE) objective of the privacy measure.

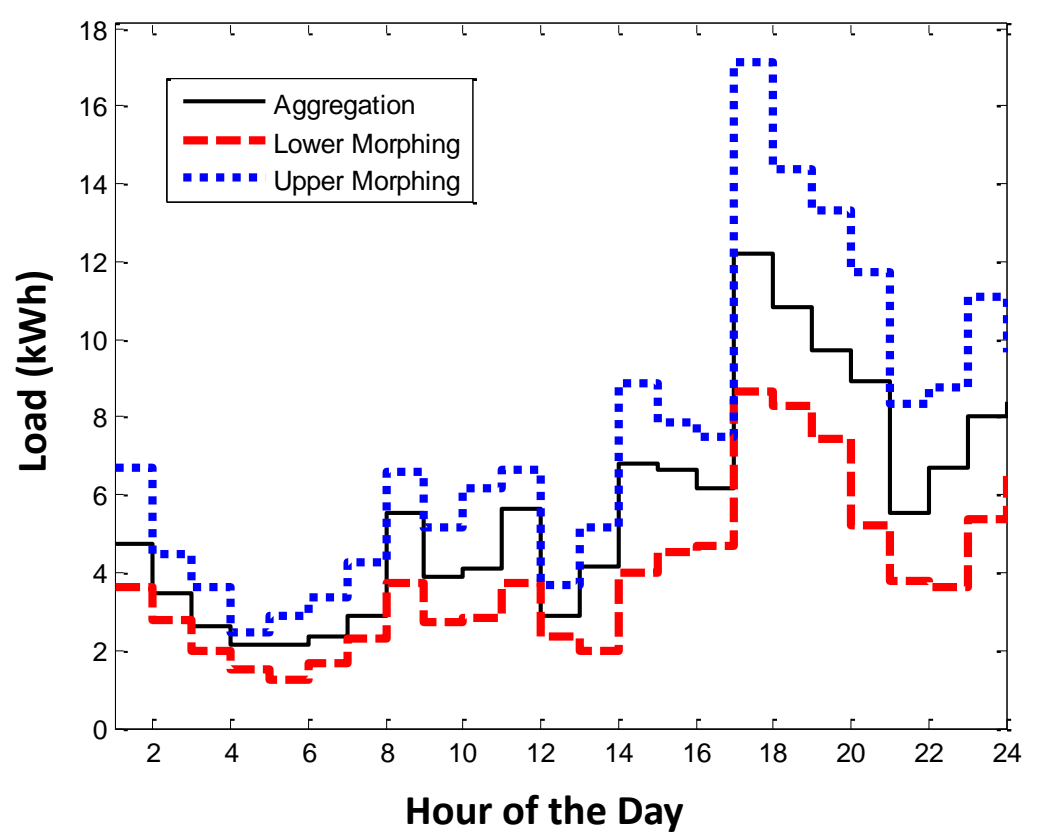

Figure 7. Aggregated load pattern of consumers 1-6 and the respective morphing upper and lower bounds.

At this point, the aggregated pattern and the morphing levels are utilized to set up the multi-objective problem, which takes the form of minimization problem. Firstly, the cost objective is defined by Equation (5), where the prices are given by Figure 4, while the load is the aggregated hourly load presented in Figure 7. Secondly, the privacy objective is given by Equation (9) where $M=0.59$, and $A_{i}^{M}$ is the aggregated hourly loads of Figure 7. Furthermore, the box constraints of the problem are taken by Equation (10) where the hourly lower and upper values are those presented in Figure 7.

Notably, the multi-objective formulation is fully defined and a solution is sought. To that end, the NSGA-II is applied to locating an optimal solution according to Pareto theory. The values of the genetic algorithm parameters, which are required to evolve the objectives, are taken as:

- Number of individuals: 30,

- Mutation probability: 0.01,

- Reproduction method: 15-point crossover,

- Max number of generations: 200,

- Selection of parents: Roulette method.

and the obtained solution is in the form of a vector whose length is equal to 24, i.e., one morphed load value for each hour of the day. The solution obtained in this specific scenario is provided in Table 1 (load values) and Figure 8 (plotted morphed pattern). We observe that the morphed aggregated 
pattern lies within the morphing bounds as determined in Figure 7 (as expected-thus validating our approach), while it differs from the initial aggregated pattern. Therefore, the algorithm has conducted a load morphing within the acceptable bounds that have been set by the grid partition consumers.

To fully assess the morphing methodology, we compute the costs and the privacy attained with the morphed pattern. In particular, we compute the following quantities:

- The non-morphed forecasted cost (NMFC), taken as the forecasted price multiplied by the aggregated load.

- The non-morphed real cost (NMRC), taken as the real price multiplied by the aggregated load.

- The morphed forecasted cost (MFC), given by the forecasted price multiplied by the morphed aggregated load.

- The morphed real cost (MRC), given by the real price multiplied by the morphed aggregated load.

- The correlation coefficient (CC) between each individual consumer pattern and the final morphed pattern denoting the degree of privacy achieved.

where we compute the costs of both forecasted and real price signals. The reason for doing that is to show that morphing the anticipated load not only reduces the anticipated cost but also the real cost.

Table 1. Load values in kWh taken as the solution of the multi-objective problem for Scenario 1.

\begin{tabular}{ccccccccccccc}
\hline Hour & $\mathbf{1}$ & $\mathbf{2}$ & $\mathbf{3}$ & $\mathbf{4}$ & $\mathbf{5}$ & $\mathbf{6}$ & $\mathbf{7}$ & $\mathbf{8}$ & $\mathbf{9}$ & $\mathbf{1 0}$ & $\mathbf{1 1}$ & $\mathbf{1 2}$ \\
\hline Solution & 4.87 & 4.10 & 3.48 & 1.53 & 1.80 & 2.77 & 4.11 & 5.60 & 2.89 & 3.04 & 3.83 & 2.54 \\
\hline Hour & $\mathbf{1 3}$ & $\mathbf{1 4}$ & $\mathbf{1 5}$ & $\mathbf{1 6}$ & $\mathbf{1 7}$ & $\mathbf{1 8}$ & $\mathbf{1 9}$ & $\mathbf{2 0}$ & $\mathbf{2 1}$ & $\mathbf{2 2}$ & $\mathbf{2 3}$ & $\mathbf{2 4}$ \\
\hline Solution & 3.11 & 4.54 & 4.74 & 5.94 & 9.21 & 8.63 & 8.09 & 5.72 & 5.66 & 4.13 & 5.46 & 6.61 \\
\hline
\end{tabular}

Morphed Aggregation

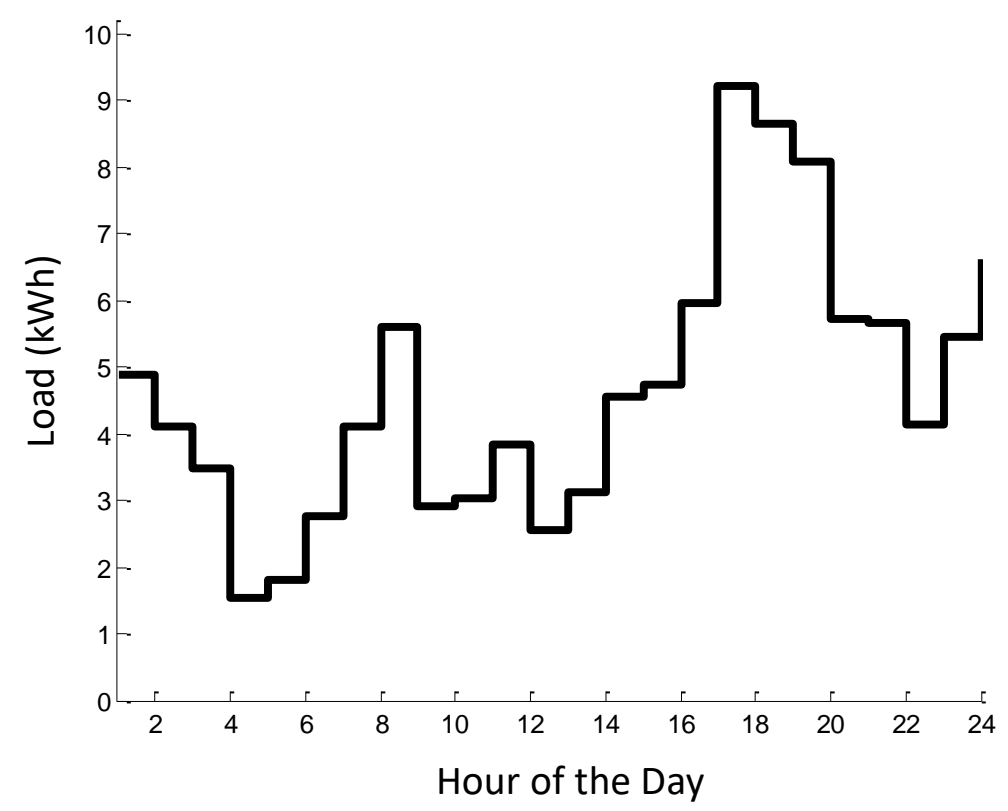

Figure 8. Plot of the final morphed aggregated load pattern computed by non-dominated sorting genetic algorithm-II (NSGA-II) (Values taken from Table 1).

Regarding the current scenario, the computed values are given in Table 2. By comparing the NMFC and the MFC, we clearly observe that the morphing methodology significantly reduced the anticipated expenses. In absolute numbers, the anticipated reduction is about $\$ 900$ as we observe in Table 2. Furthermore, by comparing the real costs we observe that indeed a reduction in the electricity cost was attained. Notably, this reduction is about $\$ 1200$, which far exceeds the anticipated reduction 
of the $\$ 900$. Thus, we conclude that by morphing the aggregated load the consumers within the grid partition attained to reduce their actual aggregated expenses-emphatically, the real expense was much lower than the anticipated one.

Table 2. Cost and privacy values computed for Scenario 1. * Values expressed in US Dollars (\$)

\begin{tabular}{ccccccc}
\hline Quantity & NMFC & MFC & NMRC & 3 & MRC & \\
\hline Value $^{*}$ & 4837 & 3978 & 6200 & & 5016 & \\
\hline Consumer & $\# \mathbf{1}$ & $\# \mathbf{2}$ & $\# \mathbf{3}$ & $\# 4$ & $\# \mathbf{5}$ & \#6 \\
\hline CC value & 0.69 & 0.41 & 0.74 & 0.75 & 0.11 & 0.26 \\
\hline
\end{tabular}

Regarding the privacy, we observe that the correlation coefficients between the initial consumers and the final morphed load has departed from the value of 1 (i.e., two patterns are fully correlated). In particular, we observe in Table 2 that the correlation values we obtain are lower than 0.76 implying that the morphed load shows less similarity to the consumers. The highest correlation is exhibited by consumers three and four whose correlation values are close to 0.75 , followed by consumer one whose value is close to 0.7 . The rest consumers exhibit very low correlation, quantified as values lower than 0.42 , with consumer five exhibiting the lowest correlation that is equal to 0.11 . Thus, we can state with much confidence that the morphed pattern differs from the individual ones, and thus masks the individual patterns. This difference, as quantified by correlation coefficient values, implies that an enhancement in each individual consumer privacy has been achieved, hence, imposing inference making of an individual's consuming activities highly challenging.

To sum up, in this first testing scenario, the six consumers via their collaboration, which is expressed in terms of load aggregation, attained to morph their initial anticipated load demand in such a way that the new patterns provides lower cost and higher privacy compared to the case of the individual non-morphed loads. This dual achievement was realized by evolving a multi-objective problem, which identified the optimal tradeoff between the cost and privacy objectives utilizing Pareto theory.

\subsection{Further Results}

In this section, the Pareto optimal morphing methodology is tested on a set of grid partitions comprised of various consumer numbers. As in the previous scenario, the data are also real-world data collected from smart meters deployed in Ireland [33]. In particular, our testing scenarios contain partitions comprised of seven (Scenario 2), 10 (Scenario 3) and 15 consumers (Scenario 4). Each test contains consumers of different load profiles, that have not been used in any of the other scenarios. Obtained results are recorded and given in Tables $3-5$ respectively. It should be noted that in our previous work, i.e., [6,29], where we had focused only on consumption morphing for privacy issues, we had showed that the values of privacy measure of groups consisted of 15 consumers and above does not significantly get improved. In particular the studies showed that the more the consumers the higher the privacy is enhanced-for values of two to 10-and above 10 consumers, we observe a plateau in the privacy value curve [29], i.e., the values exhibited no significant increase. As a result, we have selected our testing cases to include up to 15 consumers-based on our previous work [29].

Observation of the obtained results in Tables 3-5 confirms the findings of previous section. The morphing of the aggregated pattern achieves lower overall consumption cost for the grid partitions. This is something that we observe in all three studied scenarios. This decrease in cost is observed both in forecasted cost and the actual (real) cost. Therefore, the presented methodology achieved lower cost in all tested scenarios independently of the number of participating consumers, and their profiles.

For comparison purposes, we have also implemented two alternative approaches: Both approaches define single objective problems. The first approach focuses on the privacy of the partition consumers, and aims at optimizing only the privacy objective. The second approach focuses on partition's electricity 
expenses, and aims at optimizing the cost objective. It should be noted that the optimization of the single objective problems is performed with a simple genetic algorithm.

Table 3. Cost (\$) and privacy (correlation) measure values computed for Scenario 2 (Seven consumers).

\begin{tabular}{cccccc}
\hline & $\begin{array}{c}\text { NMFC } \\
\mathbf{( \$ )}\end{array}$ & $\begin{array}{c}\text { MFC } \\
\mathbf{( \$ )}\end{array}$ & $\begin{array}{c}\text { NMRC } \\
\mathbf{( \$ )}\end{array}$ & $\begin{array}{c}\text { MRC } \\
\mathbf{( \$ )}\end{array}$ & $\begin{array}{c}\text { Average Correlation } \\
\text { (between Morphed } \\
\text { and Consumers) }\end{array}$ \\
\hline $\begin{array}{c}\text { Multi Objective } \\
\text { (Our Method) }\end{array}$ & 6248 & 5077 & 8104 & 6577 & 0.427 \\
\hline $\begin{array}{c}\text { Single Objective: } \\
\text { Consumer Cost }\end{array}$ & 6248 & 5556 & 8104 & 7185 & 0.449 \\
\hline $\begin{array}{c}\text { Single Objective: } \\
\text { Consumer Privacy }\end{array}$ & 6248 & 5908 & 8104 & 7603 & 0.451 \\
\hline
\end{tabular}

Table 4. Cost (\$) and privacy (correlation) measure values computed for Scenario 3 (10 consumers).

\begin{tabular}{cccccc}
\hline & $\begin{array}{c}\text { NMFC } \\
\mathbf{( \$ )}\end{array}$ & $\begin{array}{c}\text { MFC } \\
\mathbf{( \$ )}\end{array}$ & $\begin{array}{c}\text { NMRC } \\
\mathbf{( \$ )}\end{array}$ & $\begin{array}{c}\text { MRC } \\
\mathbf{( \$ )}\end{array}$ & $\begin{array}{c}\text { Average Correlation } \\
\text { (between Morphed } \\
\text { and Consumers) }\end{array}$ \\
\hline $\begin{array}{c}\text { Multi Objective } \\
\text { (Our Method) }\end{array}$ & 6607 & 5635 & 8293 & 7039 & 0.269 \\
\hline $\begin{array}{c}\text { Single Objective: } \\
\text { Consumer Privacy }\end{array}$ & 6607 & 6068 & 8293 & 7685 & 0.286 \\
\hline $\begin{array}{c}\text { Single Objective: } \\
\text { Consumer Cost }\end{array}$ & 6607 & 6978 & 8293 & 8756 & 0.298 \\
\hline
\end{tabular}

Table 5. Cost (\$) and privacy (correlation) measure values computed for Scenario 4 (15 consumers).

\begin{tabular}{cccccc}
\hline & $\begin{array}{c}\text { NMFC } \\
\mathbf{( \$ )}\end{array}$ & $\begin{array}{c}\text { MFC } \\
\mathbf{( \$ )}\end{array}$ & $\begin{array}{c}\text { NMRC } \\
\mathbf{( \$ )}\end{array}$ & $\begin{array}{c}\text { MRC } \\
\mathbf{( \$ )}\end{array}$ & $\begin{array}{c}\text { Average Correlation } \\
\text { (between Morphed } \\
\text { and Consumers) }\end{array}$ \\
\hline $\begin{array}{c}\text { Multi Objective } \\
\text { (Our Method) }\end{array}$ & 30,421 & 25,792 & 39,385 & 33,073 & 0.327 \\
\hline $\begin{array}{c}\text { Single Objective: } \\
\text { Consumer Cost }\end{array}$ & 30,421 & 27,230 & 39,380 & 35,100 & 0.320 \\
\hline $\begin{array}{c}\text { Single Objective: } \\
\text { Consumer Privacy }\end{array}$ & 30,420 & 29,401 & 39,380 & 37,730 & 0.330 \\
\hline
\end{tabular}

By examining the computed correlation coefficients for the three scenarios, we also observe an enhancement of the consumers' privacy. In Scenario 2, all of the correlation values are lower than 0.63 , providing an average value of 0.42 . In Scenario 3, the highest correlation computed among consumers is equal to 0.8 , which implicitly exhibits that all consumers have achieved a departure from the maximum correlation value of one. In addition, we observe that the average correlation is equal to 0.26 that implicitly exhibits that the final patterns show little resemblance with the initial patterns. Lastly, in scenario 4 we observe that the all consumers with the exception of consumer 12, whose cc value is 0.87 , give correlation coefficients lower than 0.59 . Notably, the average correlation in this scenario is found to be equal to 0.32 , which also exhibits the significant departure from the max correlation of one. In sum, the morphing methodology contributes in enhancing the privacy of individual consumers; an enhancement expressed as a decrease of the correlation between the initial consumers' loads and the final morphed pattern. The average correlation per scenario is illustrative of the increased enhancement achieved by our methodology. 
In comparison with the single objective cases, we also observe that our approach achieves lower electricity cost for the partition consumers, while it retains the average correlation between consumers and the final morphed pattern at the same level or slightly lower. With regard to privacy the single objective approach (i.e., in Tables 3-5 appears as "Single Objective: Consumer Privacy") provides an average correlation that is equal to $0.45,0.29$ and 0.33 for the scenarios of seven, 10 and 15 consumers, respectively. The aforementioned correlation values are pretty close to the ones obtained with our multi-objective approach demonstrating that utilization of multiple objectives does not deteriorate the privacy driven morphing. Moreover, we observe that by focusing on privacy, the partition cost significantly increases: This is observed in Tables 3-5 with respect to MFC and MRC values, where our approach provides significantly lower costs. With regard to the cost driven approach, which optimizes the cost objective (i.e., in Tables 3-5 appears as "Single Objective: Consumer Cost"), the obtained cost values are higher than those taken with our presented multi-objective approach for all three scenarios. Hence, those results validate the ability of our approach to optimize the consumption expenses of the grid partition. Overall, the comparison of the multi-objective approach with the single objective ones exhibits the ability of our presented approach to concurrently ensure low cost and high privacy in the partition grid. Thus, we conclude that formulation of a multi-objective optimization problem that concurrently handles cost and privacy is preferable to handling each of those objectives individually, given that it provides equal or higher performance.

\section{Conclusions}

In this paper, we have presented a new methodology for morphing the load pattern of a smart power grid partition. The partitions are assemblies of consumers that exploit the smart grid communications to collaborate and pursue common goals. Their goals entail minimization of their consumption expenses as well as enhancement of their consumption privacy.

The presented methodology achieves this set of goal by formulating a multi-objective problem. The problem comprises of two objectives, namely, the cost and the privacy objective. The first objective expresses the anticipated cost consumption for a day ahead of time, and the second measures the distance of the final load pattern to the initial consumers' anticipated load. The two objectives are minimized by an evolutionary algorithm and more specifically the NSGA-II that identifies a solution using the Pareto optimality theory. Furthermore, the presented work is compared to two single objective optimization approaches: The first approach handles only the cost objective, whereas the second approach handles the privacy objective. Comparison exhibited that our approach provides equal or better performance as compared to the single objective cases. It should be noted that for the privacy measure all three approaches provided very close values, proving that our approach enhances the degree of privacy as much as the privacy objective approach does. With regard to cost, the multi-objective morphing approach provided the lowest cost values in all tested cases. By combining the above observations, we conclude that the lumping of the two objectives in a single formulation did provide a better performance as compared to single objective problems. Therefore, we conclude that NSGA-II utilizing the Pareto theory attained to concurrently secure low cost and to enhance privacy.

However, the current study exhibits some limitations. More specifically, the optimization problem is based on the box constraints for each hour of the day. In this work, we assumed narrow intervals within the morphed values may lie. In practice, these intervals may be totally different than the ones assumed in this work, and depend on the characteristics of the grid and the consumers. Furthermore, we assume that the consumers are able to form a partition via direct communication links, and that the consumers trust each other (which is not always the case). Lastly, a limitation of our study has to do with the number of customers contained in the partition: We assume that the number of partition consumers remains constant at least for a whole day (since this is the anticipation horizon).

In the current work, the morphing methodology is tested on a set of real-world data taken from smart meters deployed in the Irish power grid. Testing entails four scenarios where grid partitions are comprised of six, seven, 10 and 15 consumers. Obtained results clearly demonstrate the effectiveness 
of the methodology in decreasing the partition's consumption costs (both anticipated and real cost), and enhancing the privacy of the individual consumers. Future work will focus on identifying and expanding the set of objectives that a grid partition may pursue.

Author Contributions: M.A. developed the concept and wrote the evolutionary computing code. N.G. created the datasets. Both authors contributed in writing the paper.

Funding: This research received no external funding.

Conflicts of Interest: The authors declare no conflict of interest.

\section{References}

1. Momoh, J. Smart Grid: Fundamentals of Design and Analysis; Wiley: New York, NY, USA, 2012; Chapter 1.

2. Tsoukalas, L.H.; Gao, R. From Smart Grids to an Energy Internet: Assumptions, Architectures, and Requirements. In Proceedings of the 3rd IEEE International Conference on Electric Utility Deregulation and Restructuring and Power Technologies, Nanjing, China, 6-9 April 2008; p. 98.

3. Alamaniotis, M.; Gatsis, N.; Tsoukalas, L.H. Virtual Budget: Integration of Load and Price Anticipation for Load Morphing in Price-Directed Energy Utilization. Electr. Power Syst. Res. 2018, 158, 284-296. [CrossRef]

4. Alamaniotis, M.; Tsoukalas, L.H.; Bourbakis, N. Virtual Cost Approach: Electricity Consumption Scheduling for Smart Grids/Cities in Price-Directed Electricity Markets. In Proceedings of the IISA 2014, Chania, Greece, 7-9 July 2014; pp. 38-43.

5. Wang, W.; Xu, Y.; Khanna, M. A survey on the communication architectures in smart grid. Comput. Netw. 2011, 55, 3604-3629. [CrossRef]

6. Alamaniotis, M.; Tsoukalas, L.H.; Bourbakis, N. Anticipatory driven nodal electricity load morphing in smart cities enhancing consumption privacy. In Proceedings of the IEEE Manchester PowerTech, Manchester, UK, 18-22 June 2017; p. 6.

7. Conejo, A.J.; Carrion, M.; Morales, L.M. Decision Making Under Uncertainty in Electricity Markets; Springer: London, UK, 2010.

8. Frederiks, E.R.; Stenner, K.; Hobman, E.V. Household energy use: Applying Behavioural Economics to understand Consumer Decision-Making and Behaviour. Renew. Sustain. Energy Rev. 2015, 41, 1385-1394. [CrossRef]

9. Anjos, M.F.; Gómez, J.A. Operations Research Approaches for Building Demand Response in a Smart Grid. In Leading Developments from INFORMS Communities; INFORMS: Catonsville, MD, USA, 2017; pp. 131-152.

10. Setlhaolo, D.; Xia, X.; Zhang, J. Optimal scheduling of household appliances for demand response. Electr. Power Syst. Res. 2014, 116, 24-28. [CrossRef]

11. Lujano-Rojas, J.M.; Monteiro, C.; Dufo-López, R.; Bernal-Agustín, J.L. Optimum residential load management strategy for real time pricing (RTP) demand response programs. Energy policy 2012, 45, 671-679. [CrossRef]

12. Oprea, S.V.; Bâra, A.; Diaconita, V. Sliding Time Window Electricity Consumption Optimization Algorithm for Communities in the Context of Big Data Processing. IEEE Access 2019, 7, 13050-13067. [CrossRef]

13. Oprea, S.V.; Bâra, A.; Ifrim, G. Flattening the electricity consumption peak and reducing the electricity payment for residential consumers in the context of smart grid by means of shifting optimization algorithm. Comput. Ind. Eng. 2018, 122, 125-139. [CrossRef]

14. Gatsis, N.; Giannakis, G.B. Residential demand response with interruptible tasks: Duality and algorithms. In Proceedings of the 2011 50th IEEE Conference on Decision and Control and European Control Conference, Orlando, FL, USA, 12-15 December 2011; pp. 1-6.

15. Oprea, S.V.; Bâra, A.; Reveiu, A. Informatics solution for energy efficiency improvement and consumption management of householders. Energies 2018, 11, 138. [CrossRef]

16. Baharlouei, Z.; Hashemi, M.; Narimani, H.; Mohsenian-Rad, H. Achieving optimality and fairness in autonomous demand response: Benchmarks and billing mechanisms. IEEE Trans. Smart Grid 2013, 4, 968-975. [CrossRef]

17. Safdarian, A.; Fotuhi-Firuzabad, M.; Lehtonen, M. A distributed algorithm for managing residential demand response in smart grids. IEEE Trans. Ind. Inform. 2014, 10, 2385-2393. [CrossRef]

18. Khodaei, A.; Shahidehpour, M.; Choi, J. Optimal hourly scheduling of community-aggregated electricity consumption. J. Electr. Eng. Technol. 2013, 8, 1251-1260. [CrossRef] 
19. Verschae, R.; Kato, T.; Matsuyama, T. Energy management in prosumer communities: A coordinated approach. Energies 2016, 9, 562. [CrossRef]

20. Verschae, R.; Kawashima, H.; Kato, T.; Matsuyama, T. Coordinated energy management for inter-community imbalance minimization. Renew. Energy 2016, 87, 922-935. [CrossRef]

21. Shariatzadeh, F.; Mandal, P.; Srivastava, A.K. Demand response for sustainable energy systems: A review, application and implementation strategy. Renew. Sustain. Energy Rev. 2015, 45, 343-350. [CrossRef]

22. Chrysikou, V.; Alamaniotis, M.; Tsoukalas, L.H. A Review of Incentive based Demand Response Methods in Smart Electricity Grids. Int. J. Monit. Surveill. Technol. Res. 2015, 3, 62-73. [CrossRef]

23. Li, H.; Lin, X.; Yang, H.; Liang, X.; Lu, R.; Shen, X. EPPDR: An efficient privacy-preserving demand response scheme with adaptive key evolution in smart grid. IEEE Trans. Parallel Distrib. Syst. 2014, 25, 2053-2064. [CrossRef]

24. Gong, Y.; Cai, Y.; Guo, Y.; Fang, Y. A privacy-preserving scheme for incentive-based demand response in the smart grid. IEEE Trans. Smart Grid 2016, 7, 1304-1313. [CrossRef]

25. Wicker, S.; Thomas, R. A privacy-aware architecture for demand response systems. In Proceedings of the 44th Hawaii International Conference on System Sciences, Kauai, HI, USA, 4-7 January 2011; pp. 1-9.

26. Beligianni, F.; Alamaniotis, M.; Fevgas, A.; Tsompanopoulou, P.; Bozanis, P.; Tsoukalas, L.H. An internet of things architecture for preserving privacy of energy consumption. In Proceedings of the 10th Mediterranean Conference on Power Generation, Transmission, Distribution and Energy Conversion (Med Power 2016), Belgrade, Serbia, 6-9 November 2016; pp. 1-8.

27. Rahman, M.S.; Basu, A.; Kiyomoto, S.; Bhuiyan, M.A. Privacy-friendly secure bidding for smart grid demand-response. Inf. Sci. 2017, 379, 229-240. [CrossRef]

28. Li, D.; Aung, Z.; Williams, J.R.; Sanchez, A. No peeking: privacy-preserving demand response system in smart grids. Int. J. Parallel Emerg. Distrib. Syst. 2014, 29, 290-315. [CrossRef]

29. Alamaniotis, M.; Bourbakis, N.; Tsoukalas, L.H. Enhancing privacy of electricity consumption in smart cities through morphing of anticipated demand pattern utilizing self-elasticity and genetic algorithms. Sustain. Cities Soc. 2019, 46, 101426. [CrossRef]

30. Alamaniotis, M.; Tsoukalas, L.H.; Buckner, M. Privacy-driven electricity group demand response in smart cities using particle swarm optimization. In Proceedings of the 2016 IEEE 28th International Conference on Tools with Artificial Intelligence (ICTAI), San Jose, CA, USA, 6-8 November 2016; pp. 946-953.

31. Alamaniotis, M. Morphing to the Mean Approach of Anticipated Electricity Demand in Smart City Partitions Using Citizen Elasticities. In Proceedings of the 2018 IEEE International Smart Cities Conference (ISC2), Kansas City, MO, USA, 16-19 September 2018; pp. 1-7.

32. Alamaniotis, M.; Gatsis, N. Evolutionary Load Morphing in Smart Power System Partitions Ensuring Privacy and Minimizing Cost. In Proceedings of the 2018 Mediterranean Conference on Power Generation, Transmission, Distribution, and Energy Conversion (MEDPOWER 2018), Dubrovnik, Croatia, 12-15 November 2018; pp. 1-6.

33. Commission for Energy Regulation (CER). (2012). CER Smart Metering Project-Electricity Customer Behaviour Trial, 2009-201 [dataset], 1st ed.; Irish Social Science Data Archive: Belfield, Irland, 2007; Available online: www.ucd.ie/issda/CER-electricity (accessed on 5 March 2019).

34. Deb, K.; Pratap, A.; Agarwal, S.; Meyarivan, T.A.M.T. A fast and elitist multiobjective genetic algorithm: NSGA-II. IEEE Trans. Evol. Comput. 2002, 6, 182-197. [CrossRef]

35. Ng, Y.K. The economic theory of clubs: Pareto optimality conditions. Economica 1973, 40, 291-298. [CrossRef]

36. Eiben, A.E.; Smith, J.E. Introduction to Evolutionary Computing; Springer: Berlin/Heidelberg, Germany, 2003.

37. Pareto, V. Manuale di Economica Politica; Societa Editrice Libraria: Milan, Italy, 1906; translated by Schwier, A.S., Manual of Political Economy; Schwier, A.S., Page, A.N., Eds.; Kelley: New York, NY, USA, 1971.

38. Marler, R.; Arora, J. Survey of multi-objective optimization methods for engineering. Struct. Multidiscip. Optim. 2004, 26, 369-395. [CrossRef]

39. Nasiakou, A.; Alamaniotis, M.; Tsoukalas, L.H.; Vavalis, M. Dynamic Data Driven Partitioning of Smart Grid Using Learning Methods. In Handbook of Dynamic Data Driven Applications Systems; Springer: Berlin/Heidelberg, Germany, 2018; pp. 505-526.

40. Alamaniotis, M.; Tsoukalas, L.H. Utilization of virtual buffer in local area grids for electricity storage in smart power systems. In Proceedings of the 2017 IEEE North American Power Symposium (NAPS), Morgantown, WV, USA, 17-19 September 2017; pp. 1-6. 
41. Gao, R.; Tsoukalas, L.H. Implementing virtual buffer for electric power grids. In Proceedings of the International Conference on Computational Science, Beijing, China, 27-30 May 2007; Springer: Berlin/Heidelberg, Germany; pp. 1083-1089.

42. Alamaniotis, M.; Bargiotas, D.; Bourbakis, N.G.; Tsoukalas, L.H. Genetic optimal regression of relevance vector machines for electricity pricing signal forecasting in smart grids. IEEE Trans. Smart Grid 2015, 6, 2997-3005. [CrossRef]

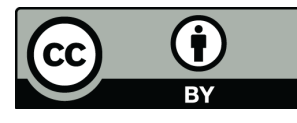

(C) 2019 by the authors. Licensee MDPI, Basel, Switzerland. This article is an open access article distributed under the terms and conditions of the Creative Commons Attribution (CC BY) license (http://creativecommons.org/licenses/by/4.0/). 